\title{
Relación entre fuerza muscular y estado de nutrición en escolares mexicanos
}

\author{
Salvador Jesús López-Alonzo, ${ }^{1, *}$ Juan Manuel Rivera-Sosa, ${ }^{1}$ Perla Zukey Hernández-Gutiérrez, ${ }^{1}$ \\ Gabriel Gastelum-Cuadras, ${ }^{1}$ Julio César Guedea-Delgado, ${ }^{1}$ Raúl Josué Nájera-Longoria ${ }^{1}$ \\ ${ }^{1}$ Facultad de Ciencias de la Cultura Física, Universidad Autónoma de Chihuahua, México.
}

\begin{abstract}
RESUMEN
Objetivo: Analizar la relación entre la fuerza muscular y el estado de nutrición en escolares entre seis y 12 años de edad. Material y métodos: Participaron 447 escolares (248 niños y 199 niñas) pertenecientes a una escuela pública estatal de la ciudad de Chihuahua, México. Edad de 8.8 \pm 1.8 años. Para medir la fuerza se utilizaron dos pruebas físicas estandarizadas: 1) flexión de brazos y 2) realización de abdominales. El estado de nutrición se evaluó por índice de masa corporal y circunferencia de cintura. Resultados: Los escolares con obesidad realizaron menos repeticiones, tanto en la prueba de flexión de brazos como en abdominales, en comparación con los estudiantes con peso normal. Se encontró una correlación inversa entre mayor sobrepeso/ obesidad con las pruebas de fuerza. Conclusiones: En escolares, la fuerza muscular se correlaciona de manera negativa con mayor sobrepeso y obesidad.
\end{abstract}

Palabras clave: Fuerza, composición corporal, escolares, salud.

\section{INTRODUCCIÓN}

La fuerza es un componente de la condición física de suma importancia en el desarrollo infantil, contribuye a una buena estructura ósea y es fundamental para el desarrollo de habilidades motrices; además, tiene

\footnotetext{
* Correspondencia: SJLA, salopez@uach.mx

Conflicto de intereses: Los autores declaran que no tienen. Citar como: López-Alonzo SJ, Rivera-Sosa JM, Hernández-Gutiérrez PZ, Gastelum-Cuadras G, Guedea-Delgado JC, NájeraLongoria RJ. Relación entre fuerza muscular y estado de nutrición en escolares mexicanos. Rev Mex Pediatr. 2019; 86(5):185-189. [Relationship between muscle strength and nutritional status in Mexican schoolchildren]
}

\begin{abstract}
Objective: To analyze the relationship between muscle strength and nutritional status in schoolchildren aged six to 12 years. Material and methods: 447 schoolchildren (248 boys and 199 girls), aged $8.8 \pm 1.8$ years, from a public school in the city of Chihuahua, Mexico participated. To measure muscle strength, two standardized physical tests were used: 1) flexion of arms and 2) performing abdominals. Nutrition status was assessed by body mass index and waist circumference. Results: Schoolchildren with obesity performed fewer repetitions, both in the arm and abdominal flexion test, compared to students with normal weight. An inverse correlation was found between greater overweight / obesity with muscle strength tests. Conclusions: In schoolchildren, muscle strength correlates negatively with overweight and obesity.
\end{abstract}

Keywords: Strength, body composition, schoolchildren, health.

funciones en el crecimiento y en el metabolismo. ${ }^{1,2}$ La condición física en los niños y adolescentes se ha convertido actualmente en un indicador de salud, ya que existe una estrecha asociación entre una mala condición física y los problemas de sobrepeso, obesidad y desnutrición. ${ }^{2,3}$

En México y en otras partes del mundo, el sobrepeso y obesidad en niños es de alto interés en la salud y educación. Según el Instituto Nacional de Salud Pública (INSP), los niños mexicanos en edad escolar muestran una prevalencia más de $30 \% .{ }^{4} \mathrm{El}$ problema de la obesidad obliga a implementar mejoras en diferentes contextos, pero es de especial interés la educación física de calidad a los niños en esta etapa de la vida. ${ }^{5-11}$ 
Para medir los componentes de la condición física se han desarrollado, validado y estandarizado diferentes pruebas físicas. Por ejemplo, en Europa se utiliza el ALPHA Fitness Test Battery para niños y adolescentes, ${ }^{10}$ y en Estados Unidos de América (EUA) el Fitness Gram, el cual es una batería de pruebas físicas que incluye la medición de la composición corporal, y fue desarrollada por el Instituto Cooper de Texas. ${ }^{11}$

En el contexto del sobrepeso/obesidad, el índice de masa corporal (IMC) es uno de los indicadores más utilizados para la evaluación del estado de nutrición, ${ }^{12}$ al igual que la circunferencia de cintura (CC). ${ }^{13,14}$

El objetivo del presente estudio fue analizar la relación entre la fuerza muscular y la composición corporal, en población escolar.

\section{MATERIAL Y MÉTODOS}

Estudio de diseño transversal y descriptivo. Fue una muestra por conveniencia, conformada por 447 escolares de nivel primaria pertenecientes a una escuela pública estatal de la ciudad de Chihuahua.

Antes de iniciar el estudio, se solicitó permiso a las autoridades correspondientes. Para su inclusión, los tutores o padres de familia firmaron carta de consentimiento informado. La participación de los escolares fue voluntaria y solamente se excluyeron quienes por restricción médica no pudieron realizar las pruebas.

Para medir la estatura (en centímetros) se utilizó un estadímetro mecánico SECA modelo 206, fijado a la pared, colocando al sujeto de espaldas y sin calzado. Para el peso (en kilogramos) se usó una báscula digital marca Tanita ${ }^{\circledR}$ modelo BF-680W, se solicitó al escolar que se colocara descalzo sobre la báscula con ropa ligera y sin calzado. Para la clasificación del estado de nutrición por IMC se utilizaron las tablas de FANTA III. ${ }^{12}$

Para medir la CC se empleó una cinta antropométrica Rosscraft ${ }^{\circledR}$; se midió por debajo de la ropa, con el escolar de pie y los brazos cruzados al pecho, se tomó de referencia el punto entre la última costilla y la cresta iliaca. Para la clasificación de CC se utilizaron las tablas de la Federación Internacional de Diabetes. ${ }^{13}$

La evaluación de la fuerza muscular fue mediante la aplicación de dos pruebas estandarizadas, las cuales forman parte del The President's Challenge Program: 1) prueba de flexión de brazos (PFB) y 2) prueba de abdominales (PABD). ${ }^{15}$

Análisis estadístico. Se realizó con el programa PASW, versión 18.0. Los datos cuantitativos se presentan con promedio y desviación estándar ( \pm DE); los cualitativos con frecuencias simples y porcentajes. La comparación de promedios fue con prueba t de Student y la correlación con r-Pearson.

\section{RESULTADOS}

Se estudiaron 248 niños y 199 niñas, con una edad de $8.8 \pm 1.8$ años. No se encontraron diferencias en cuanto a la edad y composición corporal, entre am-

\begin{tabular}{|c|c|c|c|c|c|c|c|}
\hline \multirow{2}{*}{$\begin{array}{l}\text { Variables } \\
\text { Características }\end{array}$} & \multicolumn{2}{|c|}{ Niñas $(n=199)$} & \multicolumn{2}{|c|}{ Niños $(n=248)$} & \multicolumn{2}{|c|}{ Total $(\mathrm{N}=447)$} & \multirow[b]{2}{*}{$p$} \\
\hline & Promedio & $\mathrm{DE}$ & Promedio & $\mathrm{DE}$ & Promedio & $\mathrm{DE}$ & \\
\hline Edad (años) & 8.8 & 1.7 & 8.9 & 1.8 & 8.8 & 1.8 & 0.52 \\
\hline Estatura $(\mathrm{cm})$ & 134.4 & 12.3 & 134.4 & 11.8 & 134.4 & 12.0 & 0.95 \\
\hline Peso (kg) & 34.2 & 11.4 & 34.2 & 12.0 & 34.2 & 11.7 & 0.98 \\
\hline IMC (kg/m2) & 18.6 & 4.4 & 18.5 & 4.4 & 18.5 & 4.4 & 0.88 \\
\hline Cintura $(\mathrm{cm})$ & 64.2 & 11.1 & 64.1 & 11.0 & 64.1 & 11.0 & 0.87 \\
\hline \multicolumn{8}{|l|}{ Test de fuerza } \\
\hline Flexión de brazos (repeticiones) & 1.8 & 2.6 & 5.0 & 5.7 & 3.5 & 4.9 & $<0.001$ \\
\hline Abdominales (repeticiones) & 8.9 & 6.8 & 12.18 & 8.3 & 10.7 & 7.8 & $<0.001$ \\
\hline
\end{tabular}




\begin{tabular}{lrr}
\multicolumn{3}{c}{$\begin{array}{c}\text { Tabla 2: Condición nutricia según } \\
\text { el índice de masa corporal. }\end{array}$} \\
\cline { 2 - 3 } & \multicolumn{2}{c}{ Total $(\mathrm{N}=447)$} \\
\cline { 2 - 3 } & \multicolumn{1}{c}{$\mathrm{n}$} & $\%$ \\
\hline Estado de nutrición & 3 & 0.7 \\
\hline Desnutrición grave & 52 & 11.6 \\
Desnutrición moderada & 214 & 47.9 \\
Normal & 83 & 18.6 \\
Sobrepeso & 95 & 21.3 \\
Obesidad & 178 & 39.9 \\
Sobrepeso y obesidad &
\end{tabular}

bos sexos. Pero en la fuerza muscular, los varones realizaron mayor número de repeticiones, tanto en la prueba flexión de brazos como en abdominales ( $p$ $<0.001)$ (Tabla 1).

La distribución del estado de nutrición por IMC se muestra en la Tabla 2. Además se determinó que el $41.2 \%$ de las niñas tenían la combinación de sobrepeso y obesidad, muy parecido al $38.7 \%$ de los niños. Mientras que respecto al CC (Tabla 3), más del 30\% se encontraban por arriba de la percentila 50 .

Por otra parte, al comparar la repetición de flexión de brazos y las repeticiones de abdominales con respecto al estado de nutrición (por IMC), se observó que los escolares con una condición nutricia normal hacen mayor número de repeticiones y, que en comparación con los que tienen obesidad, los que tienen sobrepeso tienen un menor desempeño (Tabla 4).

Tabla 3: Clasificación de la condición nutricia*, de acuerdo con la circunferencia de cintura.

\begin{tabular}{lccc}
$\begin{array}{lcc}\text { Estado de } \\
\text { nutrición }\end{array}$ & $\begin{array}{c}\text { Niñas } \\
(\mathrm{n}=199) \\
\%\end{array}$ & $\begin{array}{c}\text { Niños } \\
(\mathrm{n}=248) \\
\%\end{array}$ & $\begin{array}{c}\text { Total } \\
(\mathrm{N}=447) \\
\%\end{array}$ \\
\hline$\leq$ percentil 50 & 64.9 & 69.6 & 67.6 \\
$>$ percentil 50 & 7.5 & 7.3 & 7.4 \\
$>$ percentil 75 & 10.1 & 8.4 & 9.2 \\
$\geq$ percentil 90 & 17.5 & 14.5 & 15.8 \\
\hline * Clasificación Federación Internacional de Diabetes, 2007.
\end{tabular}

Lo anterior también se observó al llevar a cabo el análisis de correlación entre la fuerza y la condición nutricia. En la Tabla 5 se muestra que hay una correlación inversamente proporcional entre el valor de IMC y de CC con las repeticiones, tanto de flexión de brazos como de abdominales.

\section{DISCUSIÓN}

Los resultados del presente estudio demuestran el efecto deletéreo que existe del sobrepeso y obesidad sobre el desempeño de la fuerza muscular en escolares.

Nuestros datos son similares al estudio de Ervin, et al $2014,{ }^{8}$ realizado en niños y adolescentes de seis a 15 años de edad en EUA, donde reportan que a menor tiempo en la posición de "plancha» (prueba de fuerza) mayor es el incremento del peso corporal. El mismo estudio reveló que a medida que el peso corporal incrementa, el número de repeticiones abdominales ( $\mathrm{mo}$ dified pull-ups) disminuye. En otro trabajo realizado por Burns y Brusseau en 2017, se evaluaron 320 niños hispanos de ambos sexos de $10.1 \pm 1.1$ años de edad, en quienes se analizó la relación entre la fuerza muscular, resistencia aeróbica y síndrome metabólico. Los resultados sugieren que tanto en niños y niñas los niveles más altos de fuerza muscular y de resistencia aeróbica se relacionan con menor frecuencia de síndrome metabólico y, por ende, menor riesgo cardiometabólico. ${ }^{1}$

Asimismo, las correlaciones encontradas en el presente estudio entre la composición corporal y las pruebas de fuerza sugieren que a medida que el escolar

\section{Tabla 4: Diferencia en las pruebas de fuerza, de acuerdo con el estado de nutrición por IMC.}

\begin{tabular}{lccc} 
& \multicolumn{3}{c}{ Estado de nutrición } \\
\cline { 2 - 4 } $\begin{array}{l}\text { Pruebas de } \\
\text { fuerza }\end{array}$ & $\begin{array}{c}\text { Normal } \\
(n=214) \\
\text { Promedio }\end{array}$ & $\begin{array}{c}\text { Sobrepeso } \\
(n=95) \\
\text { Promedio }\end{array}$ & $\begin{array}{c}\text { Obesidad } \\
(n=83) \\
\text { Promedio }\end{array}$ \\
\hline $\begin{array}{l}\text { Flexión } \\
\text { de brazos } \\
\text { (repeticiones) }\end{array}$ & 4.5 & $2.8^{*}$ & $1.4^{\star *}$ \\
$\begin{array}{l}\text { Abdominales } \\
\text { (repeticiones) }\end{array}$ & 12.4 & $10.5^{\star * *}$ & $6.6^{* * * *}$ \\
\hline
\end{tabular}

* Sobrepeso vs obesidad, $p<0.001$. ${ }^{* *}$ Normal vs obesidad, $p<0.001$. ${ }^{* *}$ Normal vs sobrepeso, $p=0.03 .{ }^{* \star *}$ Normal vs obesidad, $p=0.001$. 


\begin{tabular}{|c|c|c|}
\hline & $\begin{array}{l}\text { Flexión de brazos } \\
\text { (repeticiones) }\end{array}$ & $\begin{array}{l}\text { Abdominales } \\
\text { (repeticiones) }\end{array}$ \\
\hline \multicolumn{3}{|l|}{ Niñas $(n=199)$} \\
\hline Peso corporal (kg) & $-0.226^{\star *}$ & -0.106 \\
\hline $\begin{array}{l}\text { Índice de masa } \\
\text { corporal }\left(\mathrm{kg} / \mathrm{m}^{2}\right)\end{array}$ & $-0.207^{\star *}$ & $-0.157^{\star}$ \\
\hline $\begin{array}{l}\text { Circunferencia de } \\
\text { cintura }(\mathrm{cm})\end{array}$ & $-0.282^{\star \star}$ & $-0.165^{\star}$ \\
\hline $\begin{array}{l}\text { Flexión de brazos } \\
\text { (repeticiones) }\end{array}$ & --- & $0.267^{\star *}$ \\
\hline \multicolumn{3}{|l|}{ Niños $(n=248)$} \\
\hline Peso corporal $(\mathrm{kg})$ & $-0.182^{\star *}$ & $-0.208^{\star *}$ \\
\hline $\begin{array}{l}\text { Índice de masa } \\
\text { corporal }\left(\mathrm{kg} / \mathrm{m}^{2}\right)\end{array}$ & $-0.228^{\star *}$ & $-0.299^{\star *}$ \\
\hline $\begin{array}{l}\text { Circunferencia de } \\
\text { cintura }(\mathrm{cm})\end{array}$ & $-0.193^{\star *}$ & $-0.201^{\star *}$ \\
\hline $\begin{array}{l}\text { Flexión de brazos } \\
\text { (repeticiones) }\end{array}$ & --- & $0.432^{\star *}$ \\
\hline
\end{tabular}

(tanto en las niñas como en los niños) muestra mayor CC e IMC, existe una relación negativa en el desempeño de la fuerza, puesto que disminuyen las repeticiones efectivas. Estos resultados son consistentes con los descritos por Rodríguez, et al en 2015 . $^{16}$ En dicho estudio participaron niños colombianos de ambos sexos (13.0 \pm 2.6 años de edad), y sus resultados indican que los participantes con mejor desempeño muscular presentaron también un bienestar físico más saludable con respecto a los indicadores IMC, porcentaje de grasa y circunferencia de cintura.

Los resultados de nuestro estudio pueden apoyar la necesidad de realizar intervenciones en la población de niños y adolescentes, para disminuir las comorbilidades asociadas al sobrepeso y obesidad. ${ }^{17,18}$ Se debe tomar en cuenta que el desarrollo de la fuerza en niños es de suma importancia para una buena función psicomotriz y metabólica, permitiendo al niño o adolescente conservar un sistema musculoesquelético saludable, así como un adecuado estatus de peso corporal. ${ }^{8-10,16}$

La educación física de calidad podría ser una alternativa, por poseer un enfoque adecuado e integral para formar estilos de vida saludables, junto con una buena alimentación. ${ }^{17-19}$ De esta forma, parece necesario que se desarrollen políticas intersectoriales (educación y salud), que deriven en trabajar conjuntamente con padres de familia, directivos y la comunidad en general para desarrollar programas preventivos y correctivos enfocados a mejorar la condición nutricia de escolares y adolescentes.

\section{CONCLUSIONES}

El presente estudio demuestra la relación entre la fuerza muscular y la composición corporal, evidenciando que los escolares con sobrepeso y obesidad tienen menor desempeño muscular.

\section{REFERENCIAS}

1. Burns RD, Brusseau TA. Muscular strength and endurance and cardio-metabolic health in disadvantaged Hispanic children from the U.S. Prev Med Rep. 2017; 5: 21-26.

2. Forero-Bogotá MA, Ojeda-Pardo ML, García-Hermoso A, CorreaBautista JE, Gonzalez-Jimenez E. Body composition, nutritional profile and muscular fitness affect bone health in a sample of schoolchildren from Colombia: the Fuprecol study. Nutrients. 2017; 9: 106.

3. Gray DT, Baker JS, Buchan DS. Weight status, physical activity and the associations with health related physical fitness in nine to twelve year old Scottish children. J Sports Med Doping Stud. 2013; 3: 2. doi: 10.4172/2161-0673.1000129.

4. Gutiérrez JP, Rivera-Dommarco J, Shamah-Levy T, VillalpandoHernández S, Frenco A, Cuevas-Nasu L. Resultados Nacionales de Encuesta Nacional de Salud y Nutrición. Cuernavaca, México: Instituto Nacional de Salud Pública (MX). 2012; Recuperado de: https://ensanut.insp.mx/informes/ ENSANUT2012ResultadosNacionales.pdf.

5. Centers for Disease Control and Prevention (CDC). Physical Education Curriculum Analysis Tool (PECAT). 2015; Recovered from: https://www.cdc.gov/healthyschools/pecat/index.htm.

6. Wollenstein-Seligson D, Iglesias-Leboreiro J, Bernárdez-Zapata I, Braverman-Bronstein A. Prevalencia de sobrepeso y obesidad infantil en un Hospital Privado de la Ciudad de México. Rev Mex Pediatr. 2016; 83(4): 108-114.

7. Jaramillo E. Estrategia Nacional para la Prevención y Control del Sobrepeso, obesidad y diabetes. 2017; Recuperado de: https:// www.gob.mx/cms/uploads/attachment/file/225245/2_Estrategia_ nacional_para_la_prevencion_y_control_del_sobrepeso_-DGPS.pdf.

8. Ervin RB, Fryar CD, Wang CY, Miller IM, Ogden CL. Strength and body weight in US children and adolescents. Pediatrics. 2014; 134 (6): 782-789.

9. Garcia-Marco L. Physical activity, bone mass and muscle strength in children. Acta Pediátrica. 2016; 105. 1127-1128.

10. Ruiz JR, España Romero V, Castro Piñero J, Artero EG, Ortega FB, Cuenca García, M et al. ALPHA-fitness test battery: health-related field-based fitness tests assessment in children and adolescents. Nutr Hosp. 2011; 26 (6): 1210-1214.

11. Plowman SA, Meredith MD. Fitnessgram/activitygram reference guide. 4th ed. pp. Internet Resource). Dallas, TX: The Cooper Institute. 2013; 8-1-8-55. Recuperado de: https://www. cooperinstitute.org/vault/2440/web/files/662.pdf. 
12. United States Agency for International Development (USAID), Food and Nutrition Technical Assistance. FANTA III. 2012. Recuperado de: https://www.fantaproject.org/tools/bmi-look-up-tables.

13. International Diabetes Federation (IDF). The IDF Consensus definition of the metabolic syndrome. 2007; Recuperado de: https:// www.idf.org/e-library/consensus-statements/61-idf-consensusdefinition-of-metabolic-syndrome-in-children-and-adolescents. html.

14. Kavak V, Pilmane M, Kazoka D. Body mass index, waist circumference and waist-to-hip-ratio in the prediction of obesity in Turkish teenagers. Coll Antropol. 2014; 38(2): 445-451.

15. U.S. Department of Health and Human Services, The President's Challenge is a program of the President's Council on Physical Fitness and Sports. 2010; Recuperado de: http://www.newton. k12.in.us/hs/pe/images/physical-fitness-guide.pdf.

16. Rodríguez FJ, Gualteros JA, Torres JA, Umbarila LM, RamírezVélez R. Asociación entre el desempeño muscular y el bienestar físico en niños y adolescentes de Bogotá, Colombia. Nutr Hosp. 2015; 32(4): 1559-1566.

17. Shamah-Levy T, Cuevas-Nasu L, Gaona-Pineda EB, GómezAcosta LM, Morales-Ruán MC, Hernández-Ávila M, et al. Sobrepeso y obesidad en niños y adolescentes en México, actualización de la Encuesta Nacional de Salud y Nutrición de Medio Camino 2016. Salud Pública de México. 2018; 60(3): 244-253.

18. Secretaría de Salud. Unidad de análisis económico, impacto económico del sobrepeso y la obesidad en México 19992023. 2015; Recuperado de: http://oment.uanl.mx/wp-content/ uploads/2016/09/impacto_financiero_OyS_060815_oment.pdf.

19. Organización de las Naciones Unidas para la Educación, la Ciencia y la Cultura (UNESCO). Educación Física de Calidad: Guía para responsable políticos. 2015; ISBN 97892-3-300012-4. Recuperado de: http://unesdoc.unesco.org/ images/0023/002313/231340s.pdf. 